\title{
A sensorimotor paradigm for Bayesian model selection
}

\author{
Tim Genewein ${ }^{1,2 *}$ and Daniel A. Braun ${ }^{1,2}$ \\ ${ }^{1}$ Max Planck Institute for Biological Cybernetics, Tübingen, Germany \\ ${ }^{2}$ Max Planck Institute for Intelligent Systems, Tübingen, Germany
}

\section{Edited by:}

Sven Bestmann, University College

London, UK

Reviewed by:

Amir Karniel, Ben-Gurion University,

Israel

Robert Van Beers, VU University

Amsterdam, Netherlands

\section{*Correspondence:}

Tim Genewein, Max Planck Institute

for Biological Cybernetics,

Spemannstr. 38, 72076 Tübingen,

Germany.

e-mail: tim.genewein@tuebingen. mpg.de
Sensorimotor control is thought to rely on predictive internal models in order to cope efficiently with uncertain environments. Recently, it has been shown that humans not only learn different internal models for different tasks, but that they also extract common structure between tasks. This raises the question of how the motor system selects between different structures or models, when each model can be associated with a range of different task-specific parameters. Here we design a sensorimotor task that requires subjects to compensate visuomotor shifts in a three-dimensional virtual reality setup, where one of the dimensions can be mapped to a model variable and the other dimension to the parameter variable. By introducing probe trials that are neutral in the parameter dimension, we can directly test for model selection. We found that model selection procedures based on Bayesian statistics provided a better explanation for subjects' choice behavior than simple non-probabilistic heuristics. Our experimental design lends itself to the general study of model selection in a sensorimotor context as it allows to separately query model and parameter variables from subjects.

Keywords: Bayesian model selection, sensorimotor control, structural learning, hierarchical learning, sensorimotor integration

\section{INTRODUCTION}

For biological organisms in uncertain environments, at least three important problems arise: one, the estimation of the state from noisy sensory feedback (e.g., the state of body parts). Two, the prediction of sensory consequences of actions and three, the selection of desirable actions, which builds upon the state estimate as well as the capability to predict consequences (Wolpert and Ghahramani, 2000; Todorov, 2004; Shadmehr et al., 2010; Franklin and Wolpert, 2011). Internal models are thought to play a central role in solving these problems (Shadmehr and MussaIvaldi, 1994; Wolpert et al., 1995; Kawato, 1999; Tin and Poon, 2005). For estimation, internal models make use of sensory feedback to update prior beliefs about unobserved variables. Forward models predict sensory consequences of one's own actions, which allows not only to bridge delays in the sensorimotor loop, but also to distinguish between self- and externally generated motion (Poulet and Hedwig, 2006; Imamizu, 2010). To solve the problem of action selection, the theory of optimal feedback control has been used as one of a number of frameworks that study how internal models are harnessed in control (Todorov and Jordan, 2002; Diedrichsen, 2007; Chen-Harris et al., 2008; Izawa et al., 2008; Braun et al., 2009a; Diedrichsen and Dowling, 2009; Nagengast et al., 2009).

Besides the question of how biological organisms adapt internal models when the environment changes over time, another important question is how they learn new internal models and select between existing models (Shadmehr et al., 2010). There is a large body of evidence that shows that learning of predictive models happens on many different time scales and levels of abstraction (Newell et al., 2001; Smith et al., 2006; Wolpert and Flanagan, 2010). In a number of recent studies (Braun et al., 2009a,b) it was shown, for example, that the motor system can learn structural invariants when faced with randomly changing environments that share a structural similarity. In particular, in these tasks subjects had to both adapt parameters of internal models to environments with known structure and to learn new structures and their parameters from exposure to environments with different variability pattern. Here we are interested in the mechanism by which the motor system selects between different structures, that is the selection between different models that can take on different parameter settings.

In cognitive science, a number of studies has shown that human model selection in categorization or language learning tasks can be well described as Bayesian model selection (Holyoak, 2008; Kemp and Tenenbaum, 2008; Tenenbaum et al., 2011). Bayesian models have also been very successful in explaining human perceptual and sensorimotor learning of parameters in environments with known structure (van Beers et al., 1999; Ernst and Banks, 2002; Knill and Pouget, 2004; Körding and Wolpert, 2004; Körding and Wolpert, 2006; Braun et al., 2009a; Girshick and Banks, 2009). However, if there are several structures, and each structure has a range of parameter values, then the full problem of model and parameter selection arises. For perceptual learning this has been studied, for example, in case of the ventriloquist problem, where subjects have to discriminate whether a visual and an auditory signal stem from one source or from two different sources (Körding et al., 2007; Sato et al., 2007). Here we study Bayesian model selection in the context of a sensorimotor integration task that allows for ambiguous stimuli which are compatible with different model classes. The goal of our study is to develop an experimental paradigm for model selection and to 
test whether human sensorimotor choices in such a setting are quantitatively consistent with Bayesian model selection.

\section{RESULTS}

Subjects controlled a cursor from a start position to one of two targets in a 3D virtual reality setup-see Figure 1A. At the start position the cursor was always displayed and represented subjects' veridical hand position. However, during the movement a random lateral shift $s$ was applied to the cursor with respect to the hand position. Importantly, throughout most of the movement, the cursor was hidden and there was only a brief time interval of sensory feedback of the shifted cursor position. In each trial, the shift $s$ was randomly sampled from one of two possible distributions with 50:50 probability. In the first part of the experiment (first 500 trials), the two distributions were given by a Gaussian $P\left(s \mid M_{1}^{\sigma_{1}}\right)$ and a mixture of Gaussians $P\left(s \mid M_{2}\right)$ - see Figure 2A. In the second part of the experiment (last 500 trials), the standard deviation of the first distribution was increased, so that the two distributions were given by $P\left(s \mid M_{1}^{\sigma_{2}}\right)$ and $P\left(s \mid M_{2}\right)$ - see Figure 2B.

From the point of view of model selection, the two distributions over the shift correspond to two different models $M_{1}$ and $M_{2}$. Subjects could indicate their choice of model by selecting one of the two targets. Subjects could exploit the observed shift to infer the correct target. Their belief about the shift $s$ was reported by a compensatory horizontal movement. In all trials, the upper target represented the selection of model $M_{1}$ and the lower target represented the selection of model $M_{2}$. After completing a reaching movement, participants were informed about the correctness of their beliefs by showing the shifted cursor and hiding the incorrect target. Subjects were instructed about the relationship between shift and target selection. For example, in the first part of the study they were told that small shifts are mostly associated with the upper target and larger shifts with the lower target-see "Materials and Methods" for details. They could use the first 100 trials of each part of the experiment to acquaint themselves with the decision criterion.

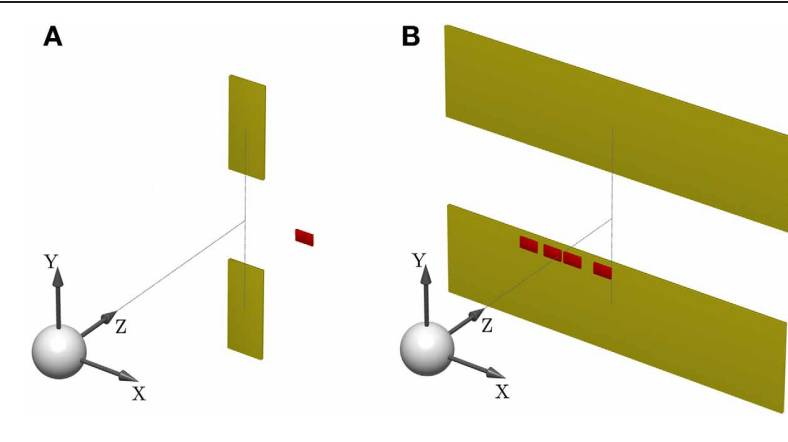

FIGURE 1 | Sensorimotor task setup. (A) Shows the schematic of a standard trial. One of two targets (yellow) has to be hit after observing a shifted cursor (red) for a short feedback duration. The white sphere depicts the start position. (B) Illustrates a probe trial where the visual feedback is ambiguous and consists of a densely sampled array of cursors, centered horizontally and vertically. The target width covers the whole lateral workspace, in order to make horizontal compensations obsolete. A photograph of the experimental apparatus is provided in Figure 15.
To test for model selection with different degrees of feedback uncertainty, we used probe trials where participants were shown ambiguous feedback. The feedback presented in these trials was an array consisting of uniformly and densely sampled rectangles that represented all the possible cursor locations-see Figure 1B. The array width $d$ could take on one of three different values with equal probability: small $(d=3 \mathrm{~cm})$, medium $(d=5 \mathrm{~cm})$, and large $(d=8 \mathrm{~cm})$. The larger the array the higher the uncertainty about the cursor position, and therefore the higher the uncertainty about the underlying shift $s$. In these probe trials subjects only reported their belief about the model without indicating the presumed shift. This was achieved by increasing the target width to the full size of the lateral workspace, which made horizontal compensations unnecessary. As in the standard trials, participants reported their belief about the correct model $M$ by choosing either the upper or the lower target, however, in probe trials they did not receive any feedback on whether their choice was correct or not. Probe trials occurred intermixed with standard trials after the first 100 trials of each part of the experiment.

In the probe trials of the first part of the experiment we found that the probability of choosing model $M_{1}^{\sigma_{1}}$ decreases with increasing width of the ambiguous feedback array: for small array widths subjects preferred model $M_{1}$, whereas for large ambiguous feedback arrays they preferred model $M_{2}$. This can be seen in Figure 3B. The correlation between the array width and the choice probability was statistically significant for all subjects ( $p<0.01$, Fisher's exact test). For the second part of the experiment, subjects' model selection probabilities are depicted in Figure 3C. The correlation between array width and choice probability was no longer significant $(p>0.1$ for all subjects, Fisher's exact test). This is because, for ambiguous feedback arrays with medium and large uncertainty $(d=5,8 \mathrm{~cm})$ subjects were now indifferent between model $M_{1}$ and $M_{2}$. Importantly, when comparing the model selection probabilities of the first part of the experiment and the second part of the experiment shown in Figure 3A, the model selection probabilities changed significantly ( $p<0.05$, ranksum test) for ambiguous stimuli with large uncertainty $(d=8 \mathrm{~cm})$. For an array width of $d=3$ or $5 \mathrm{~cm}$ there was no significant change in the choice probabilities for the two variance conditions of model $M_{1}$ ( $p>0.05$ ranksum test). Importantly, this implies that the choice probabilities of selecting model $M_{2}$ are very different for the same stimulus $(d=8 \mathrm{~cm})$ depending on the complexity of model $M_{1}$.

We tested five different explanatory schemes to describe subjects' choice behaviors: model selection with Bayes factors, model selection with Bayesian policy inference of the discrimination functions learned in the standard trials, and three heuristic explanations that are non-probabilistic.

\section{EXPLANATION 1: BAYES FACTORS}

Given prior probability $P\left(M_{i}\right)$ over the two models $M_{1}$ and $M_{2}$, Bayes' rule describes how to assign posterior probability $P\left(M_{i} \mid d\right)$ after observing array width $d$ in a probe trial, such that $P\left(M_{i} \mid d\right) \propto P\left(d \mid M_{i}\right) P\left(M_{i}\right)$-where $P\left(d \mid M_{i}\right)$ measures how well the array width $d$ can be explained on average by the shifts that are compatible with model class $M_{i}$. This average is also called the marginal likelihood or evidence and can be computed as 
A

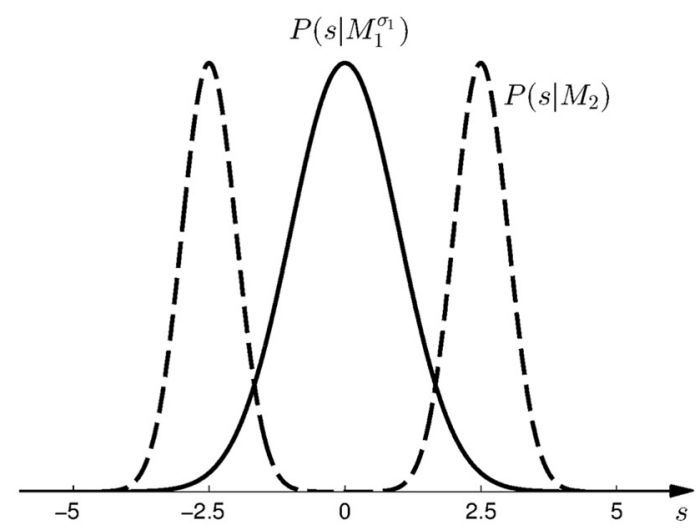

FIGURE 2 | Prior distributions for the horizontal shift $s$. (A) Shows the two prior distributions induced by model $M_{1}^{\sigma_{1}}$ (Gaussian distribution with $\sigma_{1}=1$, solid line) and $M_{2}$ (mixture of two Gaussians, dashed line) in the first
B

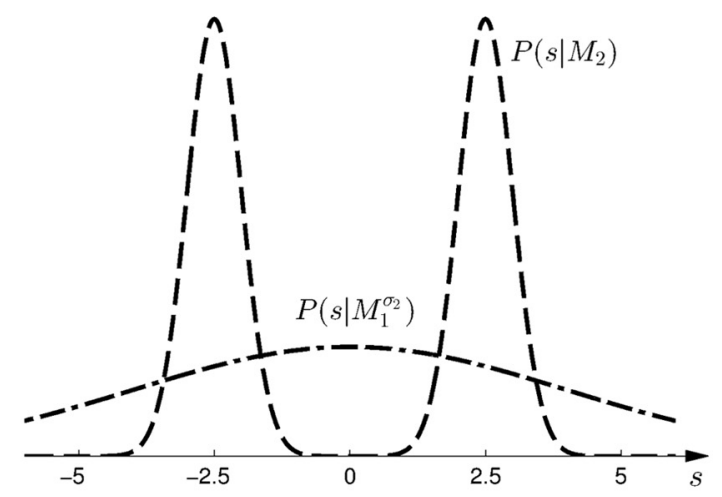

part of the study. (B) Shows the two prior distributions induced by model $M_{1}^{\sigma_{2}}$ (Gaussian distribution with $\sigma_{1}=4$, dash-dot line) and $M_{2}$ (same mixture of two Gaussians, dashed line) in the second part of the study.

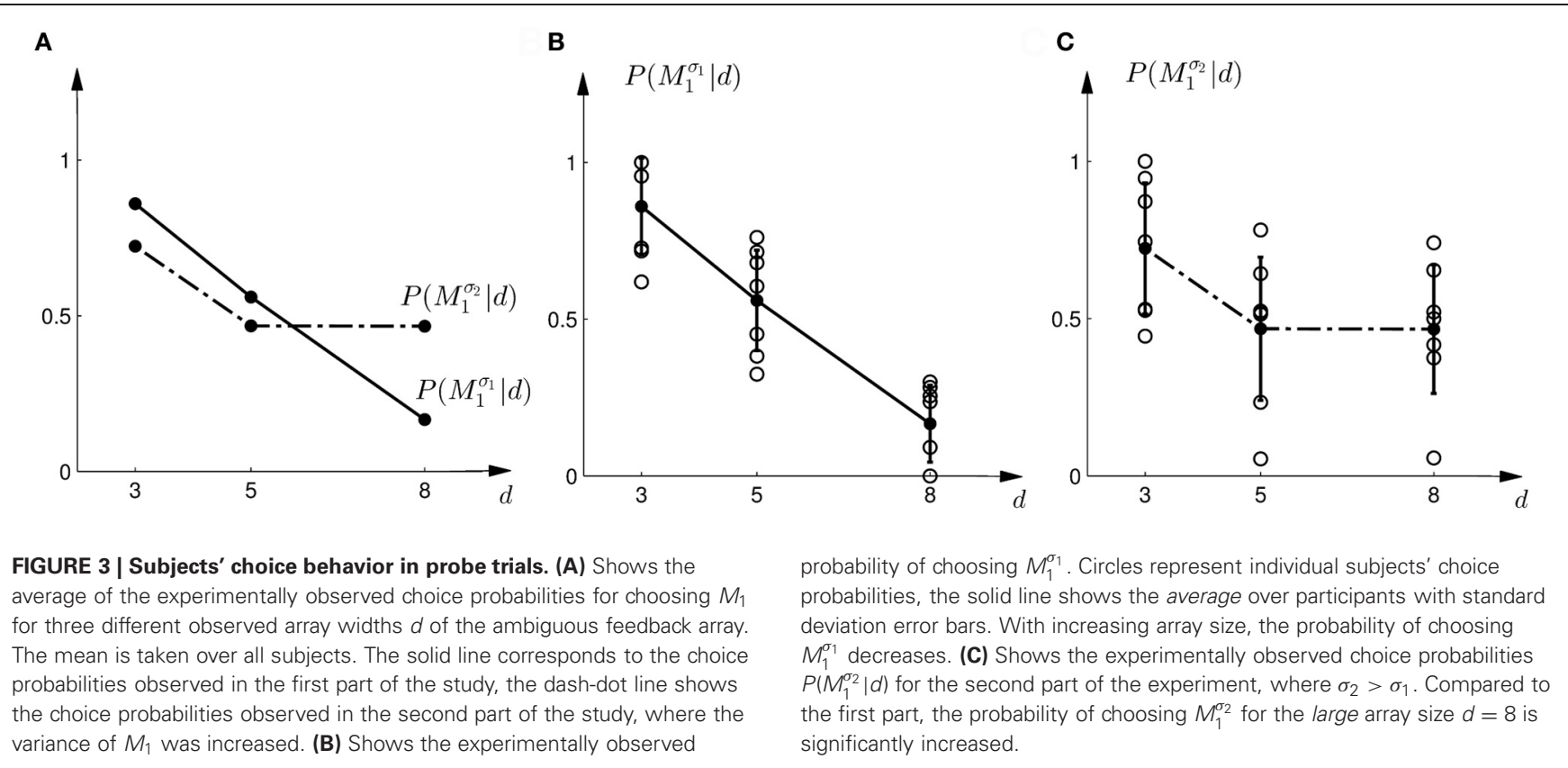

$P\left(d \mid M_{i}\right)=\int d s P\left(d \mid s, M_{i}\right) P\left(s \mid M_{i}\right)$, where each shift $s$ contributes the likelihood $P\left(d \mid s, M_{i}\right)$ weighted by the prior $P\left(s \mid M_{i}\right)$ shown in Figure 2. $P(d \mid s, M)$ is an observation model that explains how likely it is to observe an array of width $d$ if the true shift is $s$. In our experiment both models have the same observation model, that is $P(d \mid s, M)=P(d \mid s)$, which assigns equal probability to all array widths $d$ greater or equal than a given shift $s$ up to a maximum width $d_{\text {max }}$. This uniform distribution over $d$ can be seen in Figure 4A for different given shifts $s$. When $P(d \mid s)$ is used as a likelihood model, however, it is considered as a function of $s$ with a particular fixed observation $d$. The likelihood model then indicates how likely all the different shifts $s$ would be as an explanation of the observed array width $d$. The likelihood model as a function of $s$ can be seen in Figure 4B.
Based on the likelihood model in Figure 4B and the priors shown in Figure 2, Figure 5 explains how the model evidence can be computed for different observations $d$. Figure 6 shows the model evidence for the different widths $d$ of the ambiguous feedback array for all three models $M_{1}^{\sigma_{1}}, M_{1}^{\sigma_{2}}$, and $M_{2}$. As can be seen in the bottom row of Figure 6, for small width $(d=3 \mathrm{~cm})$ of the ambiguous stimulus array, the evidence for $M_{1}$ (for both $\sigma_{1}$ and $\sigma_{2}$ ) is higher than for $M_{2}$. This is because model $M_{1}$ places a high probability mass on small shifts centered around zero, whereas model $M_{2}$ does not-see Figure 2. For ambiguous feedback arrays of medium uncertainty (array width $d=5 \mathrm{~cm}$ ), the evidence of all models is very similar, that means they all can explain a medium-size range of possible shifts equally well. For ambiguous feedback arrays with large uncertainty (array width 
$d=8 \mathrm{~cm}$ ), the evidence of $M_{1}^{\sigma_{1}}$ is lower than the evidence for $M_{2}$, because model $M_{2}$ places more probability mass on larger feedback array widths, which ultimately results from the higher probability placed on large shifts—see Figure 6. However, when the standard deviation of model $M_{1}$ is increased to $\sigma_{2}$ in the second part of the study, both models can explain ambiguous feedback with large uncertainty equally well.

Once we have computed the model evidence for all possible observations and models, we can use it to make predictions about subjects' choice probabilities between the models. As we have equal prior probabilities $P\left(M_{i}\right)$ for the models in our experiment, the model class $M_{i}$ that assigns a higher marginal likelihood $P\left(d \mid M_{i}\right)$ to the observation $d$ is predicted to be preferred. Model selection is then determined by the Bayes factor (Kass and Raftery, 1993 ) between the two models, that is $P\left(d \mid M_{1}\right) / P\left(d \mid M_{2}\right)$. Based on a softmax decision rule, we can then predict subjects' choice probabilities as

$$
P\left(a=M_{1}\right)=\frac{1}{1+e^{-\alpha \log \frac{P\left(d \mid M_{1}\right)}{P\left(d \mid M_{2}\right)}}},
$$

where $a=M_{1}$ implies moving up to choose model $M_{1}$ and $a=$ $M_{2}$ implies moving down to choose model $M_{2}$. We assumed $\alpha=1$ throughout. Thus, if the Bayes factor is larger than one, subjects should be more likely to choose Model 1. Conversely, if the Bayes factor is smaller than one, subjects should be more likely to choose Model 2. The choice probabilities resulting from the softmax rule are shown in Figure 7A. In the case of small variance $\sigma_{1}$, this predicts that the probability of choosing model $M_{1}$ should decrease with the increase of the uncertainty of the ambiguous feedback. In the case of large variance $\sigma_{2}$, this predicts that the probability of choosing model $M_{1}$ is very similar to the probability of choosing model $M_{2}$ for medium and large feedback arrays. Especially for the large ambiguous feedback array of width

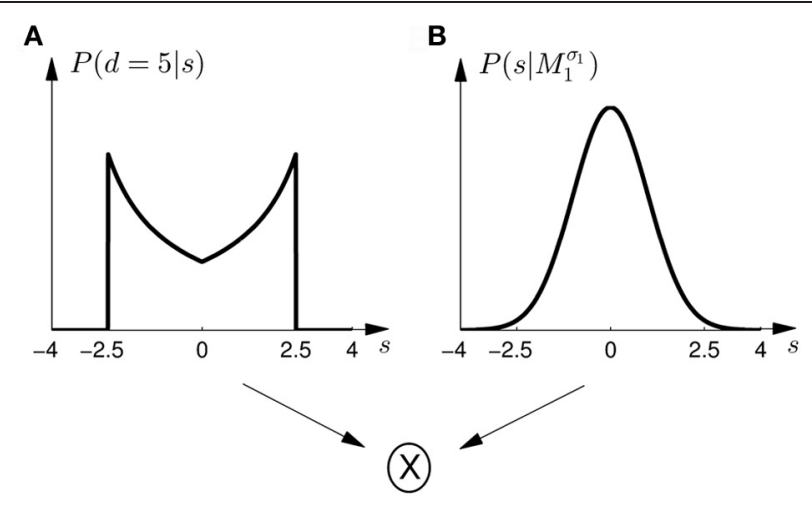

C

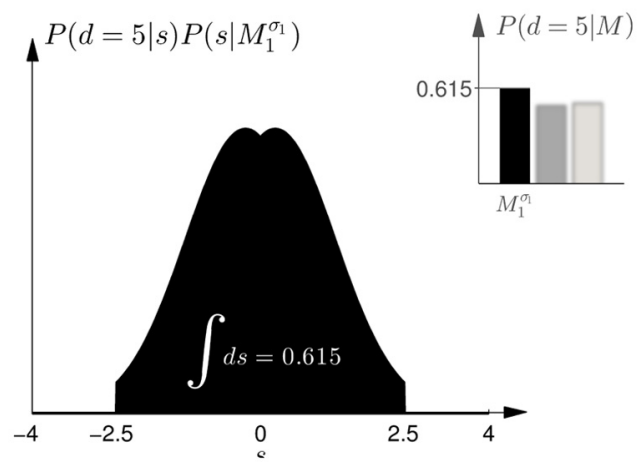

FIGURE 5 | Computation of the model evidence $P(d \mid M)$ when observing an ambiguous cursor array of width $d$-shown for model $M_{1}^{\sigma} 1$ and $d=5$, so the possible shifts of the hand position range from $\mathbf{- 2 . 5}$ to $\mathbf{2 . 5} \mathbf{c m}$. (A) Shows the likelihood $P(d=5 \mid s)$ of observing the ambiguous cursor array of width $d=5$ for different shifts $s$. (B) Shows the prior $P\left(s \mid M_{1}^{\sigma_{1}}\right)$ over the shift $s$ according to model $M_{1}^{\sigma_{1}}$. (C) Depicts the product of the distributions in panels $(\mathbf{A}, \mathbf{B})$ and illustrates the integration over the shifts $s$. This computation leads to a single value for the model evidence $P\left(d \mid M_{1}^{\sigma_{1}}\right)$. The model evidence can be similarly computed for all models as shown in the inlet panel.
A

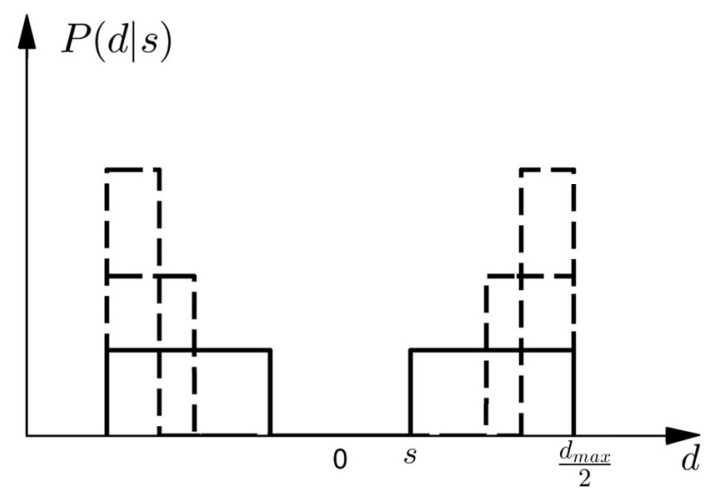

FIGURE 4 |Observation model. (A) Shows the probability $P(d \mid s)$ of observing an array of width $d$ given the shift $s$. All array widths up to a maximum $d_{\max }$ have equal probabilities but arrays that are too small to contain the shift $s$ have zero probability. The figure shows three different cases for three different shifts $s$ with a solid line and two dashed lines, respectively. It is important to notice that in

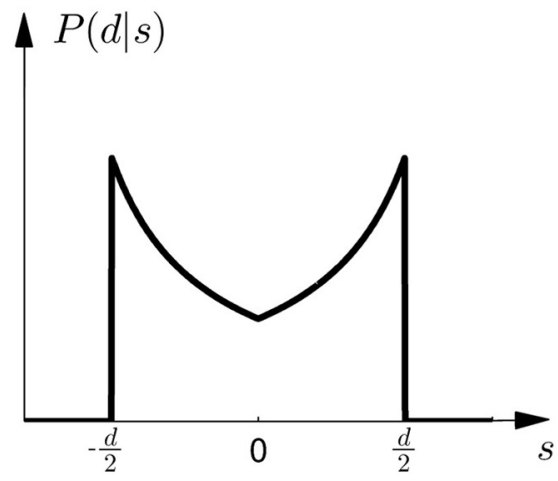

case of small shifts probability mass is spread quite evenly, whereas in the case of large shifts most of the probability mass is placed close to the maximum array width $d_{\max }$. (B) Shows the likelihood $P(d \mid s)$ as a function of $s$. For an array width $d$ it is more likely that the shift $s$ that caused the observation is close to half the array width $d / 2$. 


$$
P(d \mid s) P(s \mid M)
$$

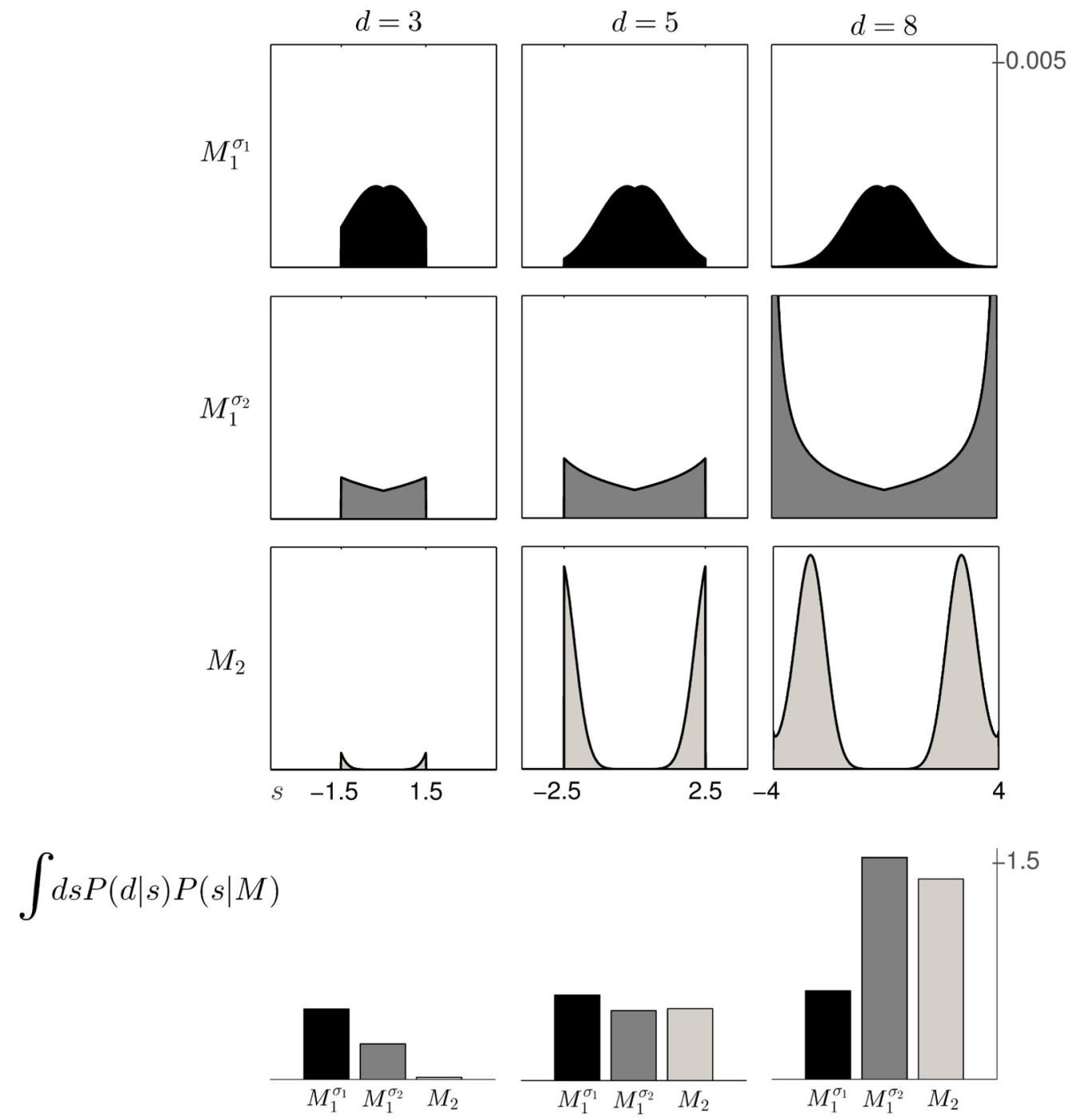

FIGURE 6 | The $3 \times 3$ panel shows the product of parameter prior $P(s \mid M)$ and observation likelihood $P(d \mid s)$ for the three possible array widths $d \in\{3,5,8\} \mathrm{cm}$ and the three different models $M_{1}^{\sigma_{1}}$ (black), $\boldsymbol{M}_{1}^{\sigma_{2}}$ (dark gray) and $\boldsymbol{M}_{\mathbf{2}}$ (light gray). The bottom row shows the model evidence $P(d \mid M)$ of observing a feedback array of width $d$ for the different models, which is obtained by integrating out the shift $s$ for each of the three models within the same column. For feedback arrays of large width $(d=8 \mathrm{~cm})$, model $M_{2}$ is a better explanation than model $M_{1}^{\sigma_{1}}$. However, when increasing the standard deviation of $M_{1}$ to $\sigma_{2}$ both models have similar marginal likelihood for large width. For a detailed illustration on the computation of the model evidence $P(d \mid M)$ see Figure 5. $d=8 \mathrm{~cm}$-the prediction implies that for the same stimulus the choice probabilities for selecting model $M_{2}$ are very different depending on the complexity of model $M_{1}$.

The comparison to the actual probabilities of model selection observed in the experiment are presented in Figures 7B,C. In line with the predictions shown in Figure 7A the probability of choosing model $M_{1}^{\sigma_{1}}$ decreases with increasing width of the ambiguous feedback array for the first part of the experiment: for small array widths subjects preferred model $M_{1}$, whereas for large ambiguous feedback arrays they preferred model $M_{2}$. Similarly, the predictions explain the choice probabilities of the probe trials in the second part of the experiment, where for small array widths model $M_{1}$ is preferred, and for larger array widths subjects are indifferent between the two models. The predictions achieved a negative $\log$-likelihood of $L=1170$ with respect to the data.

\section{EXPLANATION 2: BAYESIAN POLICY INFERENCE}

Instead of learning different prior distributions $P\left(s \mid M_{i}\right)$ over the shifts for the two models $M_{1}$ and $M_{2}$, subjects could directly learn optimal responses $P\left(a=M_{1} \mid s\right)$ to the shifts in the standard trials and a single prior $P(s)$ over the possible shifts. They could learn, for example, that for small shifts they should mostly 

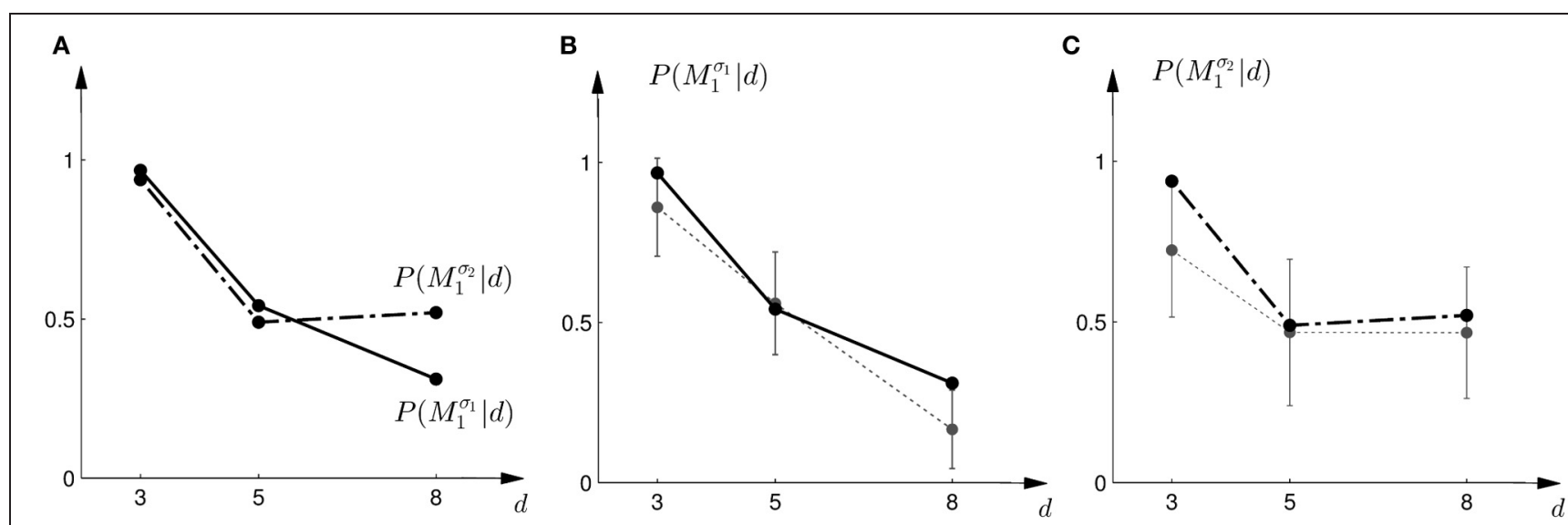

FIGURE 7 | Predictions and data: Bayes factors. (A) Shows the predicted choice probabilities for choosing $M_{1}$ as a function of the observed array width $d$ under the assumption of a softmax choice rule. The solid line corresponds to the predicted choice probabilities for the first part of the study, the dash-dot line shows the predicted choice probabilities for the second part of the study, where the variance of $M_{1}$ was increased. (B) Shows the predictions for the first part of the study (solid line) and the experimental results for three different widths $d$ of the ambiguous feedback array. The dotted line shows the mean and standard deviation across subjects of the experimentally observed probabilities of choosing $M_{1}^{\sigma_{1}}$. (C) Shows the predictions (dash-dot line) as well as the experimentally observed choice probabilities (dotted line represents mean with standard deviation error bars across subjects) for choosing $M_{1}^{\sigma_{2}}$ in the second part of the study. move to the upper target, that is $a=M_{1}$, and for large shifts they should mostly move to the lower target, that is $a=M_{2}$. When faced with an ambiguous stimulus in a probe trial, they could then integrate over the responses $P(a \mid s)$ by considering all possible shifts weighted by the plausibility of each shift. This plausibility is given by the posterior distribution $P(s \mid d)$ that results from inferring the underlying shift after observing an array of width $d$. The choice probability in the probe trial can then be computed by the integral

$$
P\left(a=M_{1} \mid d\right)=\int d s P(s \mid d) P\left(a=M_{1} \mid s\right) .
$$

This choice rule has been previously proposed as a stochastic Bayesian rule for control in (Ortega and Braun, 2010) to solve adaptive control problems. In this framework it is assumed that a number of primitive strategies are known that are suitable for different environments. When knowledge of the environment is not available a probabilistic superposition of the primitive strategies results in a stochastic strategy that conforms with Bayesian statistics. In our experiment the different environments correspond to trials with different shifts. The basic strategies coping with these shifts could be learned in the standard trials together with a prior $P(s)$ over all possible shifts. The unconditional prior $P(s)$ is given by the superposition of the two conditional priors shown in Figure 2, that is $P(s)=\frac{1}{2} P\left(s \mid M_{1}\right)+\frac{1}{2} P\left(s \mid M_{2}\right)$. In the probe trials ambiguity is induced about the underlying shift. The possible underlying shifts can be inferred through the posterior $P(s \mid d)$ that is given by $P(s \mid d) \propto P(d \mid s) P(s)$, with the same likelihood model $P(d \mid s)$ as described in the previous section and displayed in Figure 4B.

To test this model, we first investigated subjects' choice behavior in standard trials. In particular, we examined subjects' probability $P\left(a=M_{1} \mid s\right)$ of choosing model $M_{1}$ or $M_{2}$ for different shifts $s$. The response curves $P\left(a=M_{1} \mid s\right)$ for a typical subject can be seen in Figures 8A,B. Panel (A) shows the response curve for the first part of the experiment, and Panel (B) shows the response curve for the second part of the experiment. In both cases subjects showed a high probability for choosing model $M_{1}$ for small shifts. In the first part of the experiment this probability decreases for large shifts, implying the selection of model $M_{2}$. In the second part of the experiment the probability of selecting model $M_{1}$ decreases for larger shifts, but then increases again with very large shifts. These response curves are in agreement with the prior distributions shown in Figure 3, as in the first part of the experiment model $M_{1}$ was only associated with small shifts, whereas in the second part of the experiment model $M_{1}$ could also be associated with very large shifts. Learning the response functions $P(a=$ $\left.M_{1} \mid s\right)$ is therefore equivalent to learning the conditional priors $P\left(s \mid M_{i}\right)$. The fitted response curves for all subjects can be seen in Figure 9.

In the probe trials the underlying shift is unknown and therefore the policy $P\left(a=M_{1} \mid s\right)$ cannot be applied directly, as it requires knowledge of the shift $s$. Using Bayesian policy inference, the action is then determined by a probabilistic superposition that is weighted by the posterior probabilities of the shifts. This superposition allows predicting directly the choice probabilities for the probe trials. The predicted choice probabilities are shown for a typical subject in Figures 8C,D. Panel (C) shows the subject's predicted probability of choosing model $M_{1}$ for three different array widths $d$ in the first part of the experiment. It can be seen that the choice probability decreases for large observed array widths. Similarly, Panel (D) shows the subject's predicted probability of choosing model $M_{1}$ in the second part of the experiment. In this case the choice probability for model $M_{1}$ is elevated for the small array width, but is close to one half for the two larger array sizes. The comparison to the actual choice probabilities of all subjects 

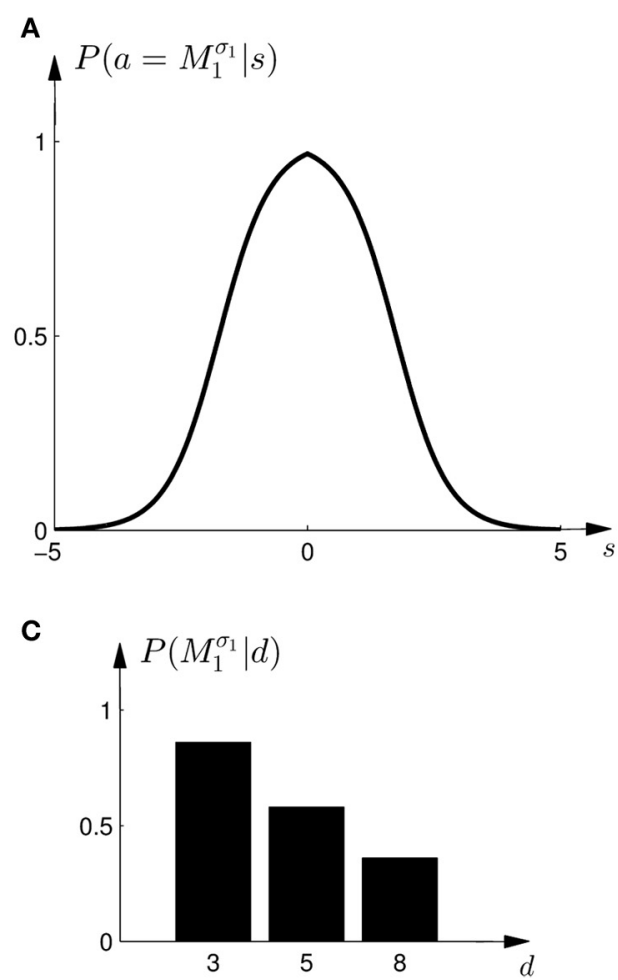

FIGURE 8 | Construction of predicted choice probabilities following Bayesian policy inference. (A) Shows the fitted choice probabilities of selecting model $M_{1}$ in standard trials when the shift $s$ is known for a typical subject in the first part of the experiment. Model $M_{1}$ is mostly selected for small shifts. (B) Shows same as panel (A) but for the
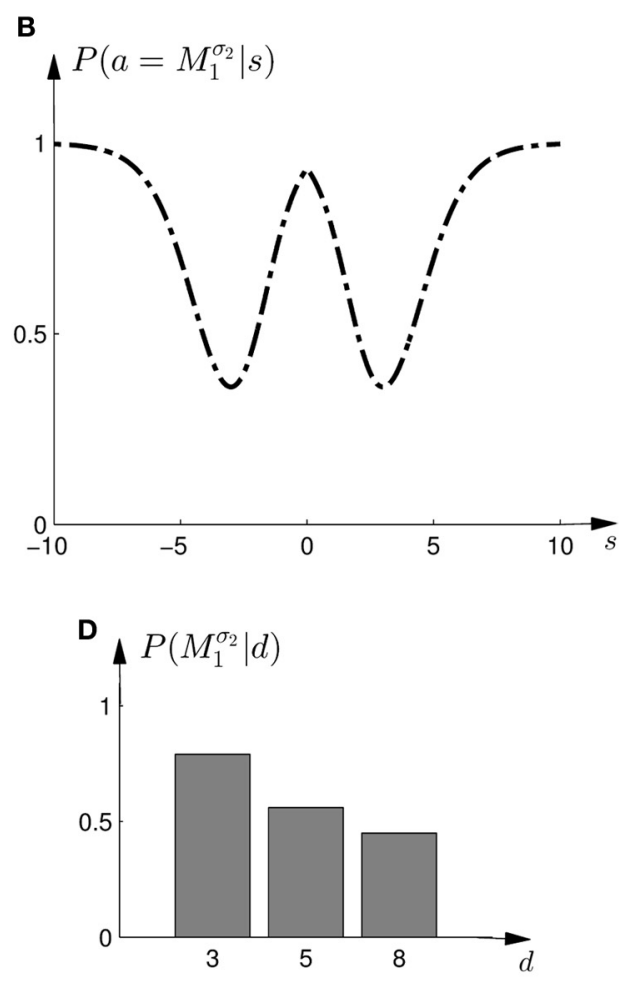

second part of the experiment. (C,D) Show the predicted choice probability of selecting model $M_{1}$ for this subject in a probe trial with observed array width $d$. The probe trial choice probabilities are obtained by a probabilistic superposition of the standard trial choice probabilities shown in panels $(\mathbf{A}, \mathbf{B})$. observed in the experiment are presented in Figure 10. The predictions achieved a total negative $\log$-likelihood of $L=1097$ with respect to the data.

\section{EXPLANATION 3: THE "AVERAGE SHIFT"-HEURISTIC}

The response curves $P(a \mid s)$, that describe behavior in the standard trials in dependence of the observed shift $s$, could also be used for non-probabilistic heuristic strategies in probe trials. One such strategy could be to simply assume the average shift when faced with an ambiguous stimulus, which corresponds to the location in the middle of the cursor array in the probe trial-see Figures 11A,B. In this case the choice probabilities are determined by

$$
P\left(a=M_{1} \mid d\right)=P\left(a=M_{1} \mid s=0\right) .
$$

However, this strategy predicts constant choice probabilities that do not vary with the observed array size. Moreover, this predicts that subjects should choose model $M_{1}$ most of the time, as it explains shifts in the middle best-see the prediction for a typical subject in Figures 11C,D. This prediction is in clear contradiction to the observed choice probabilities that change depending on the feedback array width. Figure 12 shows the predictions of the "average shift"-heuristic compared to the actual choice probabilities of all subjects. The predictions of the "average shift"-heuristic achieved a total negative $\log$-likelihood of $L=2689$ with respect to the data.

\section{EXPLANATION 4: THE "BIGGEST SHIFT"-HEURISTIC}

Another non-probabilistic heuristic that could be employed based on the response curves of the standard trials, is to assume always the largest possible shift for any given cursor array in the probe trial. Accordingly, the location of the assumed shift would correspond to the edge of the array with total width $d$, such that the edge corresponds to the half-width $d / 2$. The corresponding choice probabilities are determined by

$$
P\left(a=M_{1} \mid d\right)=P\left(a=M_{1} \mid s=d / 2\right) .
$$

The predictions of the "biggest shift"-heuristic can be seen in Figures 11E,F for a typical subject. As in the two Bayesian models, the predicted choice probability of model $M_{1}$ decreases with increasing array width for the first part of the experiment. For the second part of the experiment the "biggest shift"-heuristic predicts a slightly increased probability of choosing model $M_{1}$ for the small array width and almost indifferent choice probabilities for the two larger array widths. Figure $\mathbf{1 3}$ shows the actual choice probabilities of all subjects compared to the predictions. While the "biggest shift"-heuristic predicts the right trend in the first part of the experiments, it considerably underestimates the actual 

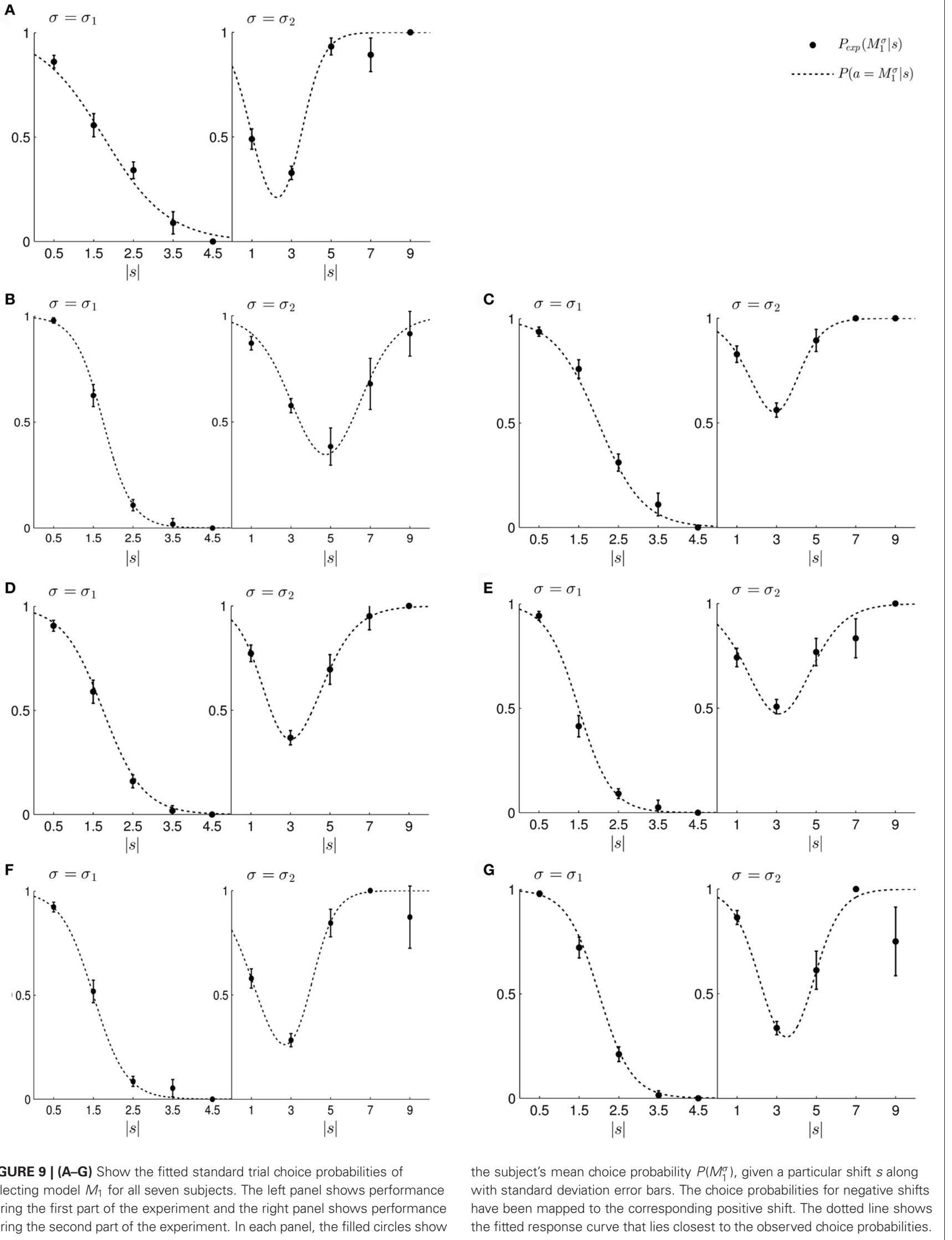

FIGURE 9 | (A-G) Show the fitted standard trial choice probabilities of selecting model $M_{1}$ for all seven subjects. The left panel shows performance during the first part of the experiment and the right panel shows performance during the second part of the experiment. In each panel, the filled circles show 


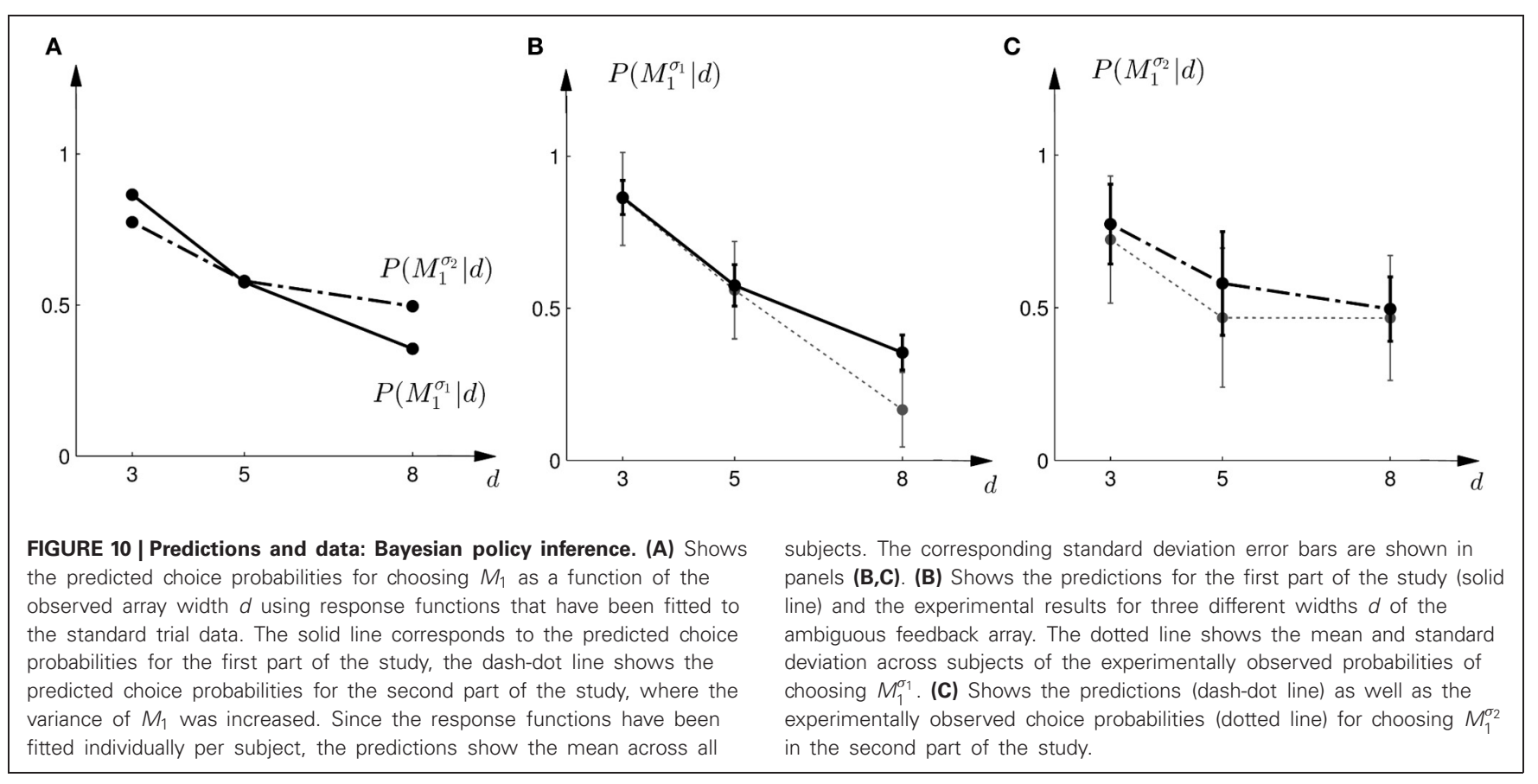

choice probabilities. The predictions for the second part of the experiment lie within the standard deviation of the experimental data. The predictions of the "biggest shift"-heuristic achieved a total negative log-likelihood of $L=1321$ with respect to the data.

\section{EXPLANATION 5: THE "HALFWAY SHIFT"-HEURISTIC}

The probe trial shifts are grossly underestimated by the "average shift"-heuristic and overestimated by the "biggest shift"-heuristic. Accordingly the choice probabilities for model $M_{1}$ are either too high or too low, especially in the first part of the experiment. We therefore considered a "halfway shift"-heuristic that would always assume a shift halfway between the middle and the edge of the cursor array. Accordingly, the choice probabilities of the "halfway shift"-heuristic lie inbetween the extremes of the other two heuristics

$$
P\left(a=M_{1} \mid d\right)=P\left(a=M_{1} \mid s=d / 4\right) .
$$

Figure 14 shows the actual choice probabilities of all subjects compared to the predictions of the "halfway shift"-heuristic. While the error bars of the experimental data and the theoretical curves overlap, it can be seen that the heuristic generally overestimates the choice probability of choosing model $M_{1}$. The predictions of the "halfway shift"-heuristic achieved a total negative $\log$-likelihood of $L=1203$ with respect to the data.

\section{DISCUSSION}

We designed a three-dimensional visuomotor integration experiment where we could distinguish between parameter variables and model variables, such that the parameter variable was represented by lateral visuomotor shifts in one dimension and the model variable was represented by two targets in the other dimension that were associated with different distributions over the shifts. In particular, we designed probe trials that did not require subjects to compensate these shifts, such that the shift variable could be "integrated out" when they reported their belief about the underlying model class. This allowed us to directly compare subjects' choice probabilities to the selection probabilities predicted by five different schemes of model selection: Bayesian model selection based on Bayes factors, Bayesian policy inference over response curves that were fitted to the standard trials, and three non-probabilistic heuristics that were also based on the standard trial response curves. We found that the Bayesian model selection procedures explained our data best, whereas the three heuristics were worse in explaining choice behavior in the probe trials. By testing two sets of distributions over the shifts, for which the observed model selection probabilities agreed with the predictions of two different Bayesian model selection procedures, we achieved a proof of concept for this experimental paradigm.

The experimental paradigm differs from previous sensorimotor paradigms on Bayesian integration (Körding and Wolpert, 2004) in two important ways. First, by introducing a third dimension to the task we can simultaneously induce uncertainty over two random variables, one of which can represent a parameter variable and the other one a model variable. Previous studies (Körding and Wolpert, 2004) have shown Bayesian integration in visuomotor tasks where only uncertainty over parameters was investigated, meaning that subjects had to infer visuomotor shifts which were drawn from a particular distribution. It was shown that subjects combined information about the prior distribution of these shifts together with noisy sensory feedback in order to obtain an optimal estimate of the shift. By varying the reliability of the sensory feedback, the authors could show that subjects weighted prior and feedback in a Bayesian way-giving less weight to the feedback if reliability of the feedback was low. As there was only one distribution over 


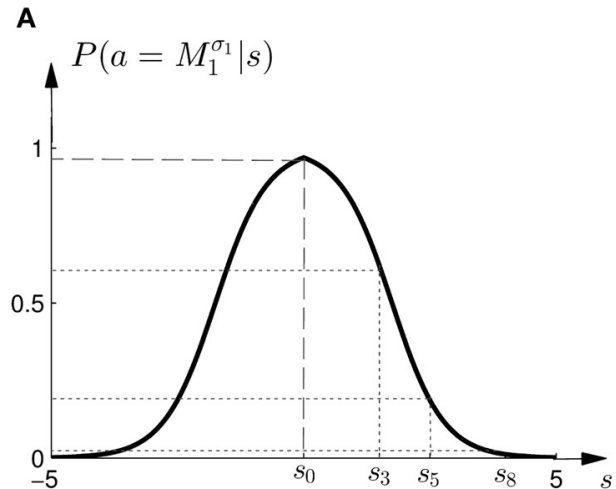

C

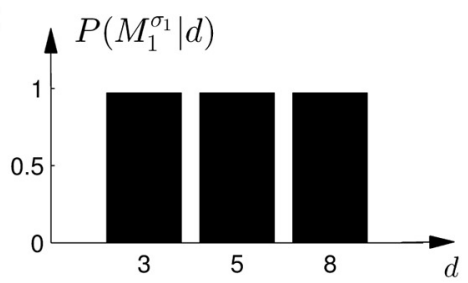

E

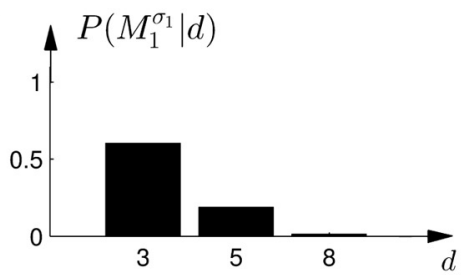

FIGURE 11 | Construction of predicted choice probabilities following heuristics. (A,B) Show the fitted choice probabilities of selecting model $M_{1}$ in standard trials of the same subject shown in Figure 8. For the "average shift"-heuristic the shift $s_{0}$ is always assumed in the probe trials. For the "biggest shift"-heuristic the shift is always assumed to be the largest possible shift that depends on the width of the feedback

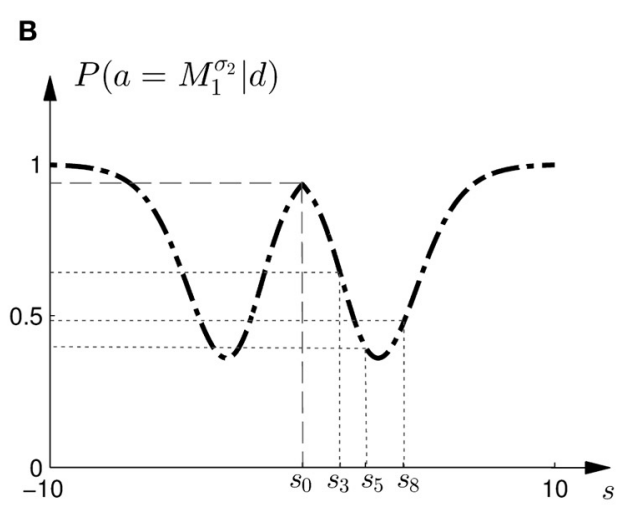

D

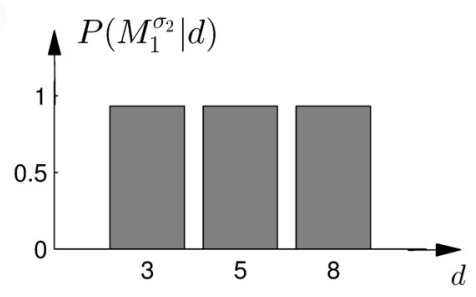

$\mathbf{F}$

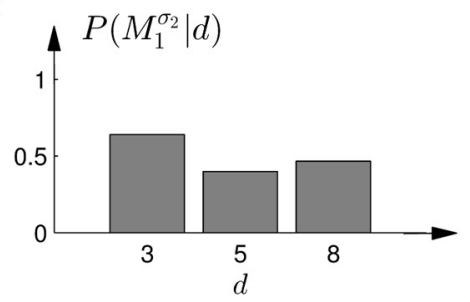

array and ranges from $s_{3}$ to $s_{8}$. (C,D) Show the predicted choice probability of selecting model $M_{1}$ for this subject in a probe trial with observed array width $d$ under the "average shift"-heuristic. (E,F) Show the predicted choice probability of selecting model $M_{1}$ for this subject in a probe trial with observed array width $d$ under the "biggest shift"-heuristic. shifts, the authors could not test for Bayesian model selection in their experiment. By introducing the third dimension for the model variable, we could therefore naturally extend their paradigm.

Second, we developed a paradigm where we can separately query the model and parameter variables from subjects. We achieved this by enlarging the horizontal size of the targets in probe trials, such that lateral corrections, that are used to report the shift parameter, become obsolete. Moreover, we clamped horizontal movements in probe trials with a force channel to ensure that only the model variable is reported. These precautions are necessary, in particular if we assume that subjects report maximum a posteriori estimates, because the maximum of a joint distribution $\max _{s, M} P(s, M \mid d)$ is not necessarily the same as the maximum of the marginal distribution $\max _{M} \sum_{s} P(s, M \mid d)$. This asymmetry is one of the most important problems when designing a sensorimotor paradigm for model selection, in which the model variable has to be queried non-verbally.
As subjects were instructed verbally at the beginning of the experiment about the relationship between the cursor shifts and the two targets, the question arises in how far cognitive processes might have played a role during the experiment. We instructed subjects about the cursor-target relationship to speed up and simplify the learning process, as we were not primarily interested in standard trial performance, but in probe trial behavior. Subjects could use these instructions as a good first guess to discriminate between the two targets. Importantly, the verbal instructions relevant for standard trials did not eliminate any of the ambiguity faced in probe trials that needed to be resolved during the movement. In this sense, our task can be conceived as a generalization of previous sensorimotor tasks (Körding and Wolpert, 2004). Nevertheless we cannot rule out that cognitive processes played a role in the perception of the ambiguous stimuli during the probe trials and the subsequent discrimination between the two models, as cognitive and sensorimotor processes are often intertwined. However, even cognitive processes have been previously shown to be consistent with Bayesian inference. 

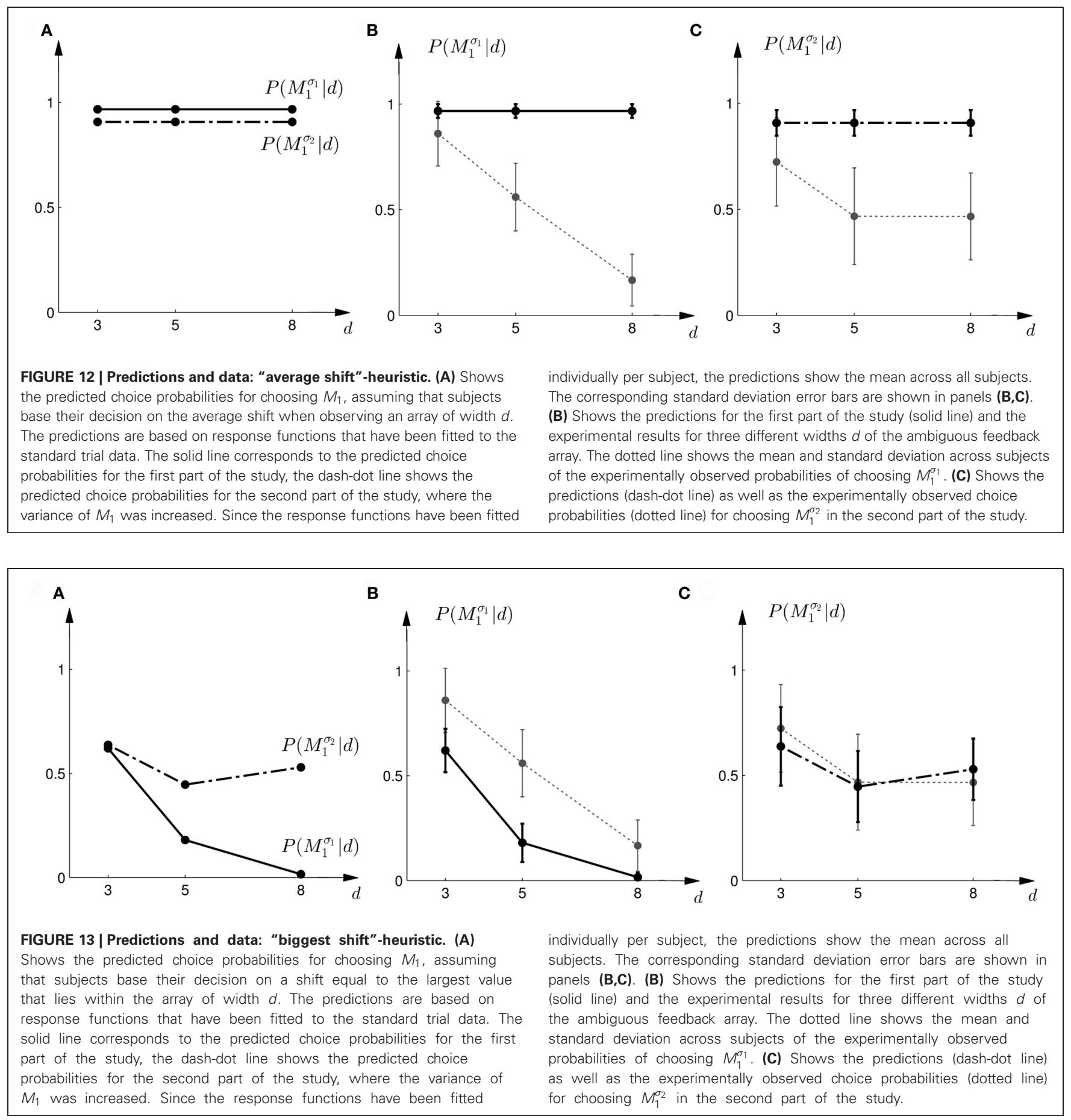

In cognitive science and perceptual learning hierarchical Bayesian inference over model classes and model parameters has been previously investigated in a number of studies (Tenenbaum et al., 2006; Körding et al., 2007; Sato et al., 2007; Holyoak, 2008; Kemp and Tenenbaum, 2008, 2009; Tenenbaum et al., 2011) In particular, (Körding et al., 2007) have studied integration vs. segregation of audio-visual stimuli in human subjects-which included inference over the two models $M_{1}$ and $M_{2}:\left(M_{1}\right)$ there is only one source for both stimuli with a location parameter $s$ and $\left(M_{2}\right)$ there are two different sources for the two stimuli with location parameters $s_{\text {visual }}$ and $s_{\text {audio }}$. To specifically look into the probabilities of model selection they modeled data from a similar previous experiment (Wallace et al., 2004), where subjects were asked to report their perception of unity. In contrast, our experimental paradigm allows for reporting model selection without the need of explicitly asking subjects verbally and without them being aware that one of the task dimensions represents a parameter variable and the other a model variable. 


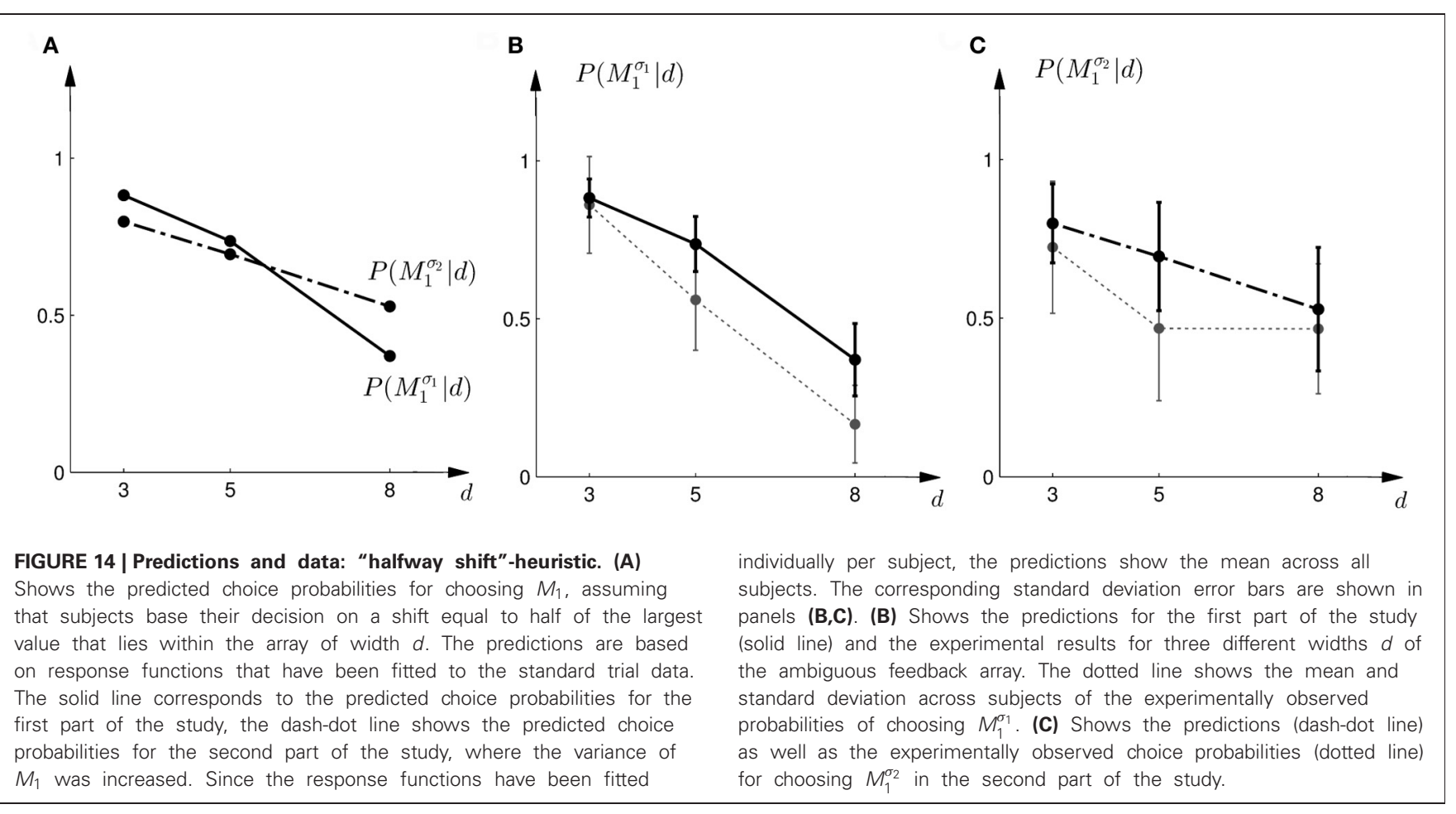

We compared five different strategies that could explain subjects' model selection probabilities in probe trials. The two Bayesian explanations had the lowest negative likelihood and therefore explained the choice probabilities in the probe trials best. However, there are important differences between the two Bayesian explanations. The first explanation explicitly computes the marginal likelihoods and uses these likelihoods as a discriminative variable. This requires the probabilistic representation of the conditional priors $P(s \mid M)$, the prior over the models $P(M)$, and the likelihood model $P(d \mid s)$. The optimal strategy is then determined over the marginal likelihood that results from an integration of these distributions. In contrast, Bayesian policy inference results from a stochastic superposition of given policies, which in our case correspond to the model selection probabilities in standard trials when the visuomotor shift is known. In probe trials, the model selection probabilities can then be determined by an integral over these standard trial policies. If subjects' choice behavior was non-stochastic we could easily distinguish between these two possibilities, as decisions based on the marginal likelihood bear no intrinsic stochasticity—we imposed it here through the softmaxfunction-and decisions resulting from the probabilistic superposition of standard trial policies would always be stochastic. Given the error bars on our data and the fact that real decisionmaking processes are always somewhat noisy, it is hard to distinguish between the two processes, even though the Bayesian policy inference achieved the lowest negative likelihood-compare Figures 7, 10.

We also examined three simple heuristics and tested in how far they might be able to explain the observed choice probabilities in probe trials. We investigated heuristics that did not consider any probabilistic representation of the task. In particular, we were investigating in how far standard trial policies could be harnessed to construct heuristics for the probe trials. A first heuristic assumed that subjects would always use the standard trial policy associated with a zero shift right in the middle of the ambiguous cursor array in the probe trial (the "average shift"heuristic). A second heuristic assumed that subjects would always use the standard trial policy associated with the largest possible shift at the edge of the ambiguous cursor array in the probe trial (the "biggest shift"-heuristic). A third heuristic was a mixture between the two, always using the standard trial policy associated with a shift halfway between the middle and the edge of the cursor array. Especially, the first two heuristics provided very poor explanations of the choice behavior, because they either systematically under- or overestimated the probability of choosing one of the models. The "halfway shift"-heuristic achieved a negative loglikelihood value that was only slightly higher than model selection with Bayes factors, but the mismatch in the fits still seemed to be systematically biased - see Figure 14. More importantly, the question remains why any of these heuristics would be formed and applied. As subjects did not receive any performance feedback in the probe trials, they could not have learned the heuristics from trial and error.

Bayesian methods are typically used in two different ways in psychophysical studies. They can simply be used as techniques to analyze the data or they can be interpreted as processes that might take place in a "Bayesian brain" that tries to make sense of the world around it. Here we used different Bayesian and nonBayesian explanations to describe subjects' choice behavior in a model selection task. This does not necessarily have any implications as to which precise algorithm the brain might use to 
achieve this behavior. In fact, there are a number of methods that have been suggested for the problem of model selection: the Akaike Information Criterion (AIC) (Akaike, 1974), the Schwarz or Bayesian Information Criterion (BIC) (Schwarz, 1978), minimum description length (MDL) (Rissanen, 1978), Bayes factors (Kass and Raftery, 1993; MacKay, 2003), structural risk minimization (Vapnik, 1995), and regularization methods (Bishop, 2006). Model selection criteria like AIC and BIC can be considered as approximations to Bayesian model selection, but also MDL, regularization and complexity measures in statistical learning theory can be related to the consideration of prior probabilities in Baysian model selection (MacKay, 2003). Finally, how model selection is achieved by neurons in the brain is subject to neurophysiological investigation.

A key capability of biological organisms is to cope with an uncertain environment. Uncertainty has many sources. It can originate from noise in the nervous system (Faisal et al., 2008), but also from uncertainty that arises in the face of ambiguous stimuli. In dealing with uncertainty, Bayesian statistics have proven to be a powerful and unifying framework not only in cognitive sciences, but also in sensorimotor tasks (Körding and Wolpert, 2006) and neural computation (Knill and Pouget, 2004; Doya, 2007; Orban and Wolpert, 2011). In particular, hierarchical Bayesian models for inference and control might allow modeling a variety of learning processes on multiple levels of abstraction (Haruno et al., 2003). Our task design provides a means to study such hierarchical integration in the context of sensorimotor control.

\section{MATERIALS AND METHODS \\ PARTICIPANTS}

Three female and four male participants were recruited from the student population of the University of Tübingen. The study was approved by the local ethics committee and all participants were naive and gave informed consent. The local standard rate of eight Euros per hour was paid for participation in the study.

\section{MATERIALS}

We used a virtual reality setup consisting of a Sensable ${ }^{\circledR}$ Phantom ${ }^{\circledR}$ Premium 1.5 High Force manipulandum for tracking participants' hand movements in three dimensions and an NVIS ${ }^{\circledR}$ nVisor ST50 head-mounted display (HMD) for creating stereoscopic 3D virtual reality-see Figure 15. Movement position and velocity were recorded with a rate of $1 \mathrm{kHz}$. To prevent very fast movements, the manipulandum was operated with a weak isotropic viscous force field of $\vec{f}=\alpha \mathbb{I}_{3 \times 3} \dot{\vec{x}}$, where $\alpha=0.04 \frac{\mathrm{Ns}}{\mathrm{cm}}, \mathbb{I}_{3 \times 3}$ is the identity and $\dot{\vec{x}}$ is the three-dimensional velocity vector.

\section{EXPERIMENTAL DESIGN}

A model selection problem can be characterized by a bivariate distribution $P(s, M)$ over a continuous random variable $s$ and a binary random variable $M$, where $s$ plays the role of the model parameter and $M$ plays the role of the model. To study model selection in a sensorimotor context, we designed a 3D visuomotor task where participants had to move a cursor from a start position to one of two targets, referred to as upper and lower

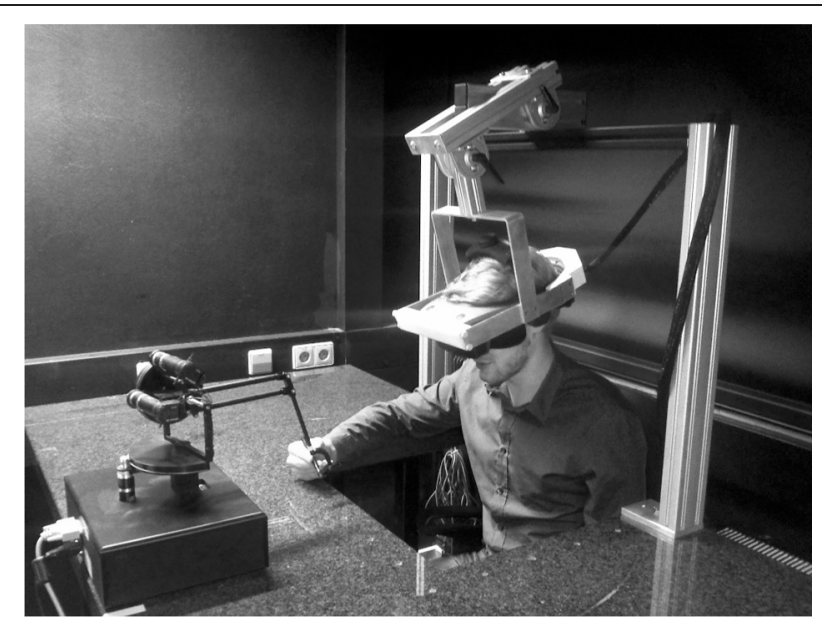

FIGURE 15 | Photograph of the experimental apparatus. The subject operates a Sensable ${ }^{\circledR}$ Phantom ${ }^{\circledR}$ Premium 1.5 High Force manipulandum and receives stereoscopic 3D visual feedback through an NVIS ${ }^{\circledR}$ nVisor ST50 head-mounted display.

target in the following-see Figure 1. During the movement, the horizontal position of the cursor was shifted, and the shift was generated by one of two possible statistical models $M \in\left\{M_{1}, M_{2}\right\}$ with 50:50 probability. Importantly, subjects were not informed about the 50:50 probability. Each target corresponded to a model $M$-the upper target corresponded to $M_{1}$ and the lower target corresponded to $M_{2}$. The correct target was the one whose corresponding model $M$ actually generated the observed shift in any particular trial. The shifts were generated by first sampling a model $M$ and then sampling a shift from the shift-distribution $P(s \mid M)$. Since shifts were generated probabilistically, any shift could in principle be generated by either model, however, with different probabilities. There was only brief sensory feedback of the shifted cursor during the movement. Participants had to use this feedback together with their knowledge of the previously learned statistical models $P(s \mid M)$ to not only infer the shift $s$, but also the model $M$. In these standard trials, participants reported their belief $P(s, M)$, where the shift $s$ was indicated by a compensatory horizontal movement when hitting a target, and the belief about the model $M$ was reported by choosing one of the two targets.

In order to test for model selection in case of feedback uncertainty, participants also experienced probe trials, in which they only reported their belief $P(M)=\int d s P(s, M)$ about the model $M$. This was achieved by increasing the width of the targets to cover the whole horizontal workspace, such that no horizontal compensatory movements were necessary in these trials. During the movement in probe trials, sensory feedback was briefly shown in shape of arrays consisting of uniformly and densely sampled rectangles that represented all the possible cursor locations-see Figure 1B. Each probe trial had one of three possible feedback array sizes (small, medium and large) that occurred equi-probably. Since the size of the feedback array constrained the uncertainty about the possible shifts $s$, Bayesian model selection required participants to "integrate out" different intervals of the 
parameter $s$ when deciding on the model $M$ by choosing either the upper or lower target.

\section{TRIAL SETUP}

Each participant performed two parts of the experiment consisting of 500 trials each. Before starting the experiment, participants were informed about the relation between observing the horizontal cursor shift and selecting one of the two targets. To start them off in the first part of the experiment, they were told that small shifts would be often associated with the upper target and larger shifts mostly with the lower target-but they were also instructed that they should use the first 100 training trials for learning this relationship precisely. Similarly, they were told for the second part of the experiment that small and very large shifts would be often associated with the upper target and medium large shifts mostly with the lower target. The initial 100 trials of each of the two sessions were standard trials only. To keep participants motivated, the hit ratio (in percent of the standard trials presented so far) was displayed. In the following 400 trials standard and probe trials were intermixed. For the probe trials, subjects were instructed that there would be a whole array of little cursors any of which could be the true cursor and that again they would have to decide which one was the correct target just like in the standard trials, but this time without knowing for sure which cursor was the correct one. The probability of presenting a probe trial was 0.45 if the previous trial was a standard trial and 0 if the previous trial was a probe trial. The second block of 500 trials was identical, only the probability distribution over shifts of model $M_{1}$ was broadened to investigate the effect on the model selection process.

\section{EXPERIMENTAL PRIOR DISTRIBUTIONS}

Each model induced a different prior probability density $P(s \mid M)$ over the horizontal shifts $s$. In part one of the study, the two models were a Gaussian and a bimodal mixture of Gaussians:

$$
\begin{aligned}
M_{1}: \quad P\left(s \mid M_{1}^{\sigma_{1}}\right)= & \mathcal{N}\left(0,1 \mathrm{~cm}^{2}\right) \\
M_{2}: \quad P\left(s \mid M_{2}\right)= & \frac{1}{2} \mathcal{N}\left(-2.5 \mathrm{~cm}, 0.25 \mathrm{~cm}^{2}\right) \\
& +\frac{1}{2} \mathcal{N}\left(2.5 \mathrm{~cm}, 0.25 \mathrm{~cm}^{2}\right) .
\end{aligned}
$$

In part two of the study, the same distributions were used, only the standard deviation of $P\left(s \mid M_{1}\right)$ was increased such that

$$
\begin{aligned}
M_{1}: \quad P\left(s \mid M_{1}^{\sigma_{2}}\right)= & \mathcal{N}\left(0,16 \mathrm{~cm}^{2}\right) \\
M_{2}: \quad P\left(s \mid M_{2}\right)= & \frac{1}{2} \mathcal{N}\left(-2.5 \mathrm{~cm}, 0.25 \mathrm{~cm}^{2}\right) \\
& +\frac{1}{2} \mathcal{N}\left(2.5 \mathrm{~cm}, 0.25 \mathrm{~cm}^{2}\right) .
\end{aligned}
$$

The prior probability of both models was always $P\left(M_{1}\right)=$ $P\left(M_{2}\right)=\frac{1}{2}$. A plot of the prior distributions is shown in Figure 3 .

\section{EXPERIMENTAL PROCEDURE: STANDARD TRIALS}

After hearing a beep, participants initiated a reaching movement by controlling a cursor (red sphere, radius $0.4 \mathrm{~cm}$ ) from a start position (gray sphere, semi-transparent, radius $0.9 \mathrm{~cm}$ ) to one of two target blocks (yellow cuboids, height $5 \mathrm{~cm}$, width $2 \mathrm{~cm}$ )—see Figure 1A. One of the target blocks was in the upper half of the workspace, the other target block was in the lower half-with a distance of $2 \mathrm{~cm}$ in-between. Both target blocks were presented at a depth of $18.5 \mathrm{~cm}$ with respect to the start position. Once the cursor had left the start position, it was invisible and an additive random shift was applied to the cursor position. The shift was drawn from a distribution $P(s \mid M)$ once $M$ had been sampled from $P(M)$. The correct target was determined by the sampled $M$, that is the upper target was correct if $M=M_{1}$ and the lower target was correct if $M=M_{2}$. While the cursor was invisible during the movement, after a movement depth of $5.5 \mathrm{~cm}$ visual feedback (red rectangle, width $0.8 \mathrm{~cm}$, height $0.3 \mathrm{~cm}$ ) of the shifted cursor position was displayed for $100 \mathrm{~ms}$. When the movement exceeded a depth of $18.5 \mathrm{~cm}$ the trial ended. If the cursor was in-between the two targets without touching either of them, the trial continued until one of the targets was chosen. For hitting a target, the cursor had to at least touch the target block. When participants hit the correct target, a high-pitch beep was played. When participants hit the wrong target or missed the correct target, a low-pitch beep was played. In either case the incorrect target disappeared. At the end of the reach, the shifted cursor position was shown. If movement was still in progress after $2 \mathrm{~s}$, the trial was aborted and had to be repeated.

\section{EXPERIMENTAL PROCEDURE: PROBE TRIALS}

In contrast to standard trials, the target width of the two targets was increased to $20 \mathrm{~cm}$ in probe trials, thereby covering the entire horizontal workspace. Crucially, this made any compensatory movements in the horizontal direction obsolete and reduced the task to a binary model selection problem that only required choosing either the upper or lower target. To further discourage horizontal compensatory movements in probe trials, we generated a "force tunnel" that did not allow left/right deviations from the middle of the workspace, but only up/down and forward/backward movements. Since sideward movements were not necessary in probe trials, the impact of the tunnel force was barely noticeable and most participants reported that they did not notice it at all when interviewed after the experiment.

Probe trials also started with a beep, after which participants initiated a reaching movement to one of the two targets (yellow cuboids, height $5 \mathrm{~cm}$, width $20 \mathrm{~cm}$ ) - see Figure 1B. At a movement depth of $5.5 \mathrm{~cm}$ visual feedback was displayed for $100 \mathrm{~ms}$. However, in contrast to the standard trials, feedback was not shown as a little rectangle representing the shifted hand position, but as an array of multiple same-sized rectangles that were sampled simultaneously and uniformly from one of three possible horizontal intervals: $[-1.5,1.5],[-2.5,2.5]$, and $[-4.0,4.0] \mathrm{cm}$. The little rectangles had $0.8 \mathrm{~cm}$ width and there were 4,7 , and 10 little rectangles, respectively shown for small, medium and large bar size at any one time frame. The probability of showing one of the array sizes was one third. Participants were informed that this array indicated all possible cursor positions and that the true cursor was at one of the many possible positions seen in the array. Since sideward deviations were impossible due to the tunnel force and vertical deviations carried no information with 
respect to possible shifts, the arrays were always centered in the workspace both horizontally and vertically. In order to make participants understand that the shift was sampled uniformly from the cursor array, at the end of the movement, after participants had chosen one of the two targets, a cursor position was drawn from the uniform distribution over the shown interval and displayed. However, no visual or auditive feedback was given to indicate whether the correct target was hit or not. The three possible widths for the interval (small: $3 \mathrm{~cm}$, medium: $5 \mathrm{~cm}$, and large: $8 \mathrm{~cm}$ ) induced an increasing amount of uncertainty about possible shifts. In the probe trials we could therefore investigate how these different amounts of uncertainty with respect to the parameter $s$ affected the selection of the model $M$, when the parameter $s$ was not reported and therefore could be "integrated out."

\section{MODELING}

For the two Bayesian explanations of choice behavior in probe trials, we used the following observation model. Crucially, in our experiment observing an array of width $d \geq 0$ is the same for both models $M_{1}$ and $M_{2}$ and therefore the likelihood model only depends on the shift variable $s$, such that

$$
P(d \mid s)= \begin{cases}\frac{1}{\frac{d_{\max }}{2}-|s|} & \text { if } d \geq 2|s| \text { and } d \leq d_{\max } \\ 0 & \text { otherwise, }\end{cases}
$$

where $d_{\max }$ represents the maximum possible array width. In our experiment $d_{\max }=8 \mathrm{~cm}$. This observation model implies that for any given shift $s$, the array size cannot be smaller than $s$, since it must contain $s$, and the array size cannot exceed the maximum size $d_{\max }$. All array sizes in-between have equal probability. After observing array size $d$, Bayes' rule allows us to infer the posterior over both the shift and the model

$$
P(s, M \mid d)=\frac{P(d \mid s) P(s \mid M) P(M)}{\sum_{M} \int d s P(d \mid s) P(s \mid M) P(M)} .
$$

\section{REFERENCES}

Akaike, H. (1974). A new look at the statistical model identification. IEEE T. Autom. Control AC-19, 716-723.

Bishop, C. M. (2006). Pattern Recognition and Machine Learning, Vol. 4. New York, NY: Springer.

Braun, D. A., Aertsen, A., Wolpert, D. M., and Mehring, C. (2009a). Learning optimal adaptation strategies in unpredictable motor tasks. J. Neurosci. 29, 6472-6478.

Braun, D. A., Aertsen, A., Wolpert, D. M., and Mehring, C. (2009b). Motor task variation induces structural learning. Curr. Biol. 19, 352-357.

Chen-Harris, H., Joiner, W. M., Ethier, V., Zee, D. S., and Shadmehr, R. (2008). Adaptive control of saccades via internal feedback. J. Neurosci. 28, 2804-2813.

Diedrichsen, J. (2007). Optimal taskdependent changes of bimanual

Curr. Biol. 17, 1675-1679.

The Bayes factor can be derived from this posterior by realizing that $P(d \mid M)=P(M \mid d)$ and $P(M \mid d)=\int d s P(s, M \mid d)$. In case of the Bayesian policy inference the posterior $P(s \mid d)$ can be derived from the joint posterior by realizing that $P(s \mid d)=\sum_{M} P(s, M \mid d)$.

In line with Bayesian policy inference the posterior $P(s \mid d)$ is used for the probabilistic superposition of the standard trial policies $P\left(a=M_{1} \mid s\right)$ such that the choice probability in probe trials is given by $P\left(a=M_{1} \mid d\right)=\int d s P(s \mid d) P\left(a=M_{1} \mid s\right)$. The standard trial policies $P(a \mid s)$ were fitted to the data as follows. First, all standard trials were sorted into five equidistant bins depending on the magnitude of the shift in each trial. For the first part of the experiment the five bins were $[0,1],[1,2],[2,3],[3,4]$, and $[4,5] \mathrm{cm}$. For the second part of the experiment the five bins were $[0,2],[2,4],[4,6],[6,8]$, and $[8,10] \mathrm{cm}$. The relative frequencies of choosing model $M_{1}$ in these bins was fitted by a sigmoid psychometric function

$$
P\left(a=M_{1} \mid s\right)=1-\frac{1}{1+e^{\frac{-s+\zeta}{\theta}}}
$$

for the first part of the experiment and a two-partite sigmoid function

$$
P\left(a=M_{1} \mid s\right)=1-\frac{1}{1+e^{\frac{-s+\gamma}{\delta}}}+\frac{1}{1+e^{\frac{-s+\kappa}{\tau}}}
$$

for the second part of the experiment. The free parameters $\zeta, \theta, \gamma, \delta, \kappa, \tau$ were fitted by minimizing square error. The fits can be seen in Figure 9.

\section{ACKNOWLEDGMENTS}

This study was supported by the DFG, Emmy Noether grant BR4164/1-1.

Diedrichsen, J., and Dowling, N. (2009). Bimanual coordination as taskdependent linear control policies. Hum. Mov. Sci. 28, 334-347.

Doya, K. (ed.). (2007). Bayesian Brain: Probabilistic Approaches to Neural Coding. Cambridge, MA, USA: MIT Press.

Ernst, M. O., and Banks, M. S. (2002). Humans integrate visual and haptic information in a statistically optimal fashion. Nature 415, 429-433.

Faisal, A. A., Selen, L. P. J., and Wolpert, D. M. (2008). Noise in the nervous system. Nat. Rev. Neurosci. 9, 292-303.

Franklin, D. W., and Wolpert, D. M. (2011). Computational mechanisms of sensorimotor control. Neuron 72, 425-442.

Girshick, A. R., and Banks, M. S. (2009). Probabilistic combination

Imamizu, H. (2010). Prediction of of slant information: weighted averaging and robustness as optimal percepts. J. Vis. 9, 8.1-820.

Kawato, M. (1999). Internal models for motor control and trajectory planning. Curr. Opin. Neurobiol. 9, 718-727.

Haruno, M., Wolpert, D. M., and Kawato, M. (2003). Hierarchical mosaic for movement generation. Int. Cong. Ser. 1250, 575-590.

Holyoak, K. J. (2008). Induction as model selection. Proc. Natl. Acad. Sci. U.S.A. 105, 10637-10638. sensorimotor feedback from the efference copy of motor commands: a review of behavioral and functional neuroimaging studies. Jpn. Psych. Res. 52, 107-120.

Izawa, J., Rane, T., Donchin, O., and Shadmehr, R. (2008). Motor adaptation as a process of reoptimization. J. Neurosci. 28, 2883-2891.

Kass, R. E., and Raftery, A. E. (1993). Bayes factors and model uncertainty. J. Am. Stat. Assoc. 90, 466.
Kemp, C., and Tenenbaum, J. B. (2008). The discovery of structural form. Proc. Natl. Acad. Sci. U.S.A. 105, 10687-10692.

Kemp, C., and Tenenbaum, J. B. (2009). Structured statistical models of inductive reasoning. Psychol. Rev. 116, 20-58.

Knill, D. C., and Pouget, A. (2004). The bayesian brain: the role of uncertainty in neural coding and computation. Trends Neurosci. 27, 712-719.

Körding, K. P., Beierholm, U., Ma, W. J., Quartz, S., Tenenbaum, J. B., and Shams, L. (2007). Causal inference in multisensory perception. PLoS ONE 2:e943. doi:10.1371/journal.pone.0000943

Körding, K. P., and Wolpert, D. M. (2004). Bayesian integration in 
sensorimotor learning. Nature 427, 244-247.

Körding, K. P., and Wolpert, D. M. (2006). Bayesian decision theory in sensorimotor control. Trends Cogn. Sci. 10, 319-326.

MacKay, D. J. C. (2003). Information heory, Inference and Learning Algorithms. Cambridge, UK: Cambridge University Press.

Nagengast, A. J., Braun, D. A., and Wolpert, D. M. (2009). Optimal control predicts human performance on objects with internal degrees of freedom. PLoS Comput. Biol. 5:e1000419. doi:10.1371/journal.pcbi.1000419

Newell, K. M., Liu, Y. T., and Mayer-Kress, G. (2001). Time scales in motor learning and development. Psychol. Rev. 108, 57-82.

Orban, G., and Wolpert, D. M. (2011). Representations of uncertainty in sensorimotor control. Curr. Opin. Neurobiol. 21, $1-7$.

Ortega, P. A., and Braun, D. A. (2010). A minimum relative entropy principle for learning and acting. J. Artif. Intell. Res. 38, 475-511.

Poulet, J. F. A., and Hedwig, B. (2006). The cellular basis of a corollary discharge. Science 311, 518-522.
Rissanen, J. (1978). Modeling by shortest data description. Automatica 14, 465-471.

Sato, Y., Toyoizumi, T., and Aihara, K. (2007). Bayesian inference explains perception of unity and ventriloquism aftereffect: identification of common sources of audiovisual stimuli. Neural Comput. 19, 3335-3355.

Schwarz, G. (1978). Estimating the dimension of a model. Ann. Stat. 6 , 461-464.

Shadmehr, R., and Mussa-Ivaldi, F. (1994). Adaptive representation of dynamics during learning of a motor task. J. Neurosci. 14, 3208-3224.

Shadmehr, R., Smith, M. A., and Krakauer, J. W. (2010). Error correction, sensory prediction, and adaptation in motor control. Annu. Rev. Neurosci. 33, $89-108$.

Smith, M. A., Ghazizadeh, A., and Shadmehr, R. (2006). Interacting adaptive processes with different timescales underlie short-term motor learning. PLoS Biol. 4:e179. doi:10.1371/journal.pbio.0040179

Tenenbaum, J. B., Griffiths, T. L. and Kemp, C. (2006). Theory-based bayesian models of inductive learning and reasoning. Trends Cogn. Sci. 10, 309-318.
Tenenbaum, J. B., Kemp, C., Griffiths, T. L., and Goodman, N. D. (2011). How to grow a mind: statistics, structure, and abstraction. Science 331, 1279-1285.

Tin, C., and Poon, C.-S. (2005) Internal models in sensorimotor integration: perspectives from adaptive control theory. J. Neural Eng. 2, S147.

Todorov, E. (2004). Optimality principles in sensorimotor control. Nat. Neurosci. 7, 907-915.

Todorov, E., and Jordan, M. I. (2002) Optimal feedback control as a theory of motor coordination. Nat. Neurosci. 5, 1226-1235.

van Beers, R. J., Sittig, A. C., and Gon, J. J. (1999). Integration of proprioceptive and visual positioninformation: an experimentally supported model. J. Neurophysiol. 81, 1355-1364.

Vapnik, V. N. (1995). The Nature of Statistical Learning Theory, Vol. 8 . New York, NY: Springer.

Wallace, M. T., Roberson, G. E., Hairston, W. D., Stein, B. E. Vaughan, J. W., and Schirillo, J. A. (2004). Unifying multisensory signals across time and space. Exp. Brain Res. 158, 252-258

Wolpert, D. M., and Flanagan, J. R. (2010). Motor learning. Curr. Biol. 20, R467-R472.
Wolpert, D. M., and Ghahramani, Z. (2000). Computational principles of movement neuroscience. Nat Neurosci. 3(Suppl.), 1212-1217.

Wolpert, D. M., Ghahramani, Z., and Jordan, M. I. (1995). An internal model for sensorimotor integration. Science 269, 1880-1882.

Conflict of Interest Statement: The authors declare that the research was conducted in the absence of any commercial or financial relationships that could be construed as a potential conflict of interest.

Received: 01 August 2012; accepted: 03 October 2012; published online: 19 October 2012.

Citation: Genewein $T$ and Braun DA (2012) A sensorimotor paradigm for Bayesian model selection. Front. Hum. Neurosci. 6:291. doi: 10.3389/fnhum. 2012.00291

Copyright (c) 2012 Genewein and Braun. This is an open-access article distributed under the terms of the Creative Commons Attribution License, which permits use, distribution and reproduction in other forums, provided the original authors and source are credited and subject to any copyright notices concerning any third-party graphics etc. 